Chirurg 2012 $\cdot 83: 916$

DOI 10.1007/s00104-012-2368-9

Online publiziert: 20. September 2012

(c) Springer-Verlag Berlin Heidelberg 2012

O. Strobel · M.W. Büchler

Klinik für Allgemein-, Viszeral- und Transplantationschirurgie, Universität Heidelberg

\title{
Langzeitergebnisse nach Gallenwegs- und vaskulobiliären Verletzungen im Rahmen einer Cholezystektomie
} Cholecystectomy-related bile duct and vasculobiliary injuries. Br J Surg 99:1129-1136

\section{Hintergrund und Fragestellung}

Bei etwa 0,2-0,5\% aller Cholezystektomien kommt es als Komplikation zu Gallenwegsverletzungen. Bei bis zu 60\% dieser Fälle liegt eine kombinierte Gefäßund Gallenwegsverletzung (vaskulobiliäre Verletzung, VBV) vor. Während isolierte Gefäßverletzungen meist kompensiert werden und unerkannt bleiben, stellt die VBV eine schwere Komplikation mit oft komplexem Verlauf dar.

Sarno et al. analysierten bei Patienten mit Gallenwegsverletzungen Langzeitergebnisse und Risikofaktoren für ein schlechtes Langzeit-Outcome.

\section{Methoden}

Es wurden 63 Patienten (45 isolierte schwere Gallenwegsverletzungen [Strasberg E], $18 \mathrm{VBV}$ ) identifiziert, die zwischen 1990 und 2010 nach extern erfolgter Cholezystektomie an das Zentrum der Autoren verlegt wurden. Patienten mit Zystikusstumpfinsuffizienz und isolierten Gefäßverletzungen wurden ausgeschossen. Zur Rekonstruktion der Gallengangsläsionen erfolgte bei allen Patienten eine End-zu-Seit-Roux-y-Hepatikojejunostomie. Die Patienten wurden nach 1, 3, 6 und 12 Monaten und dann jährlich nachkontrolliert. Als gutes Outcome wur- den das Fehlen einer den Gallenwegen oder der Rekonstruktion zuzuordnenden Symptomatik und nicht mehr als 2-fach erhöhte Leberwerte definiert.

paraturversuch waren keine Risikofaktoren für ein schlechtes Outcome.

\section{Fazit}

\section{Ergebnisse}

Von den 63 Patienten wurden 40 bei starkem Galleleck, 6 bei Gallengangsstriktur, 15 bei rezidivierenden Cholangitiden und 2 wegen starker intraoperativer Blutung 20 Tage (Median) nach Cholezystektomie überwiesen. Bei 24 Patienten wurde die Verletzung intraoperativ, bei $39 \mathrm{~Pa}$ tienten (62\%) erst 8 Tage (Median) postoperativ erkannt. Bei 28 Patienten (61\%) war vor Verlegung bereits ein chirurgischer Reparaturversuch erfolgt: meist durch End-zu-End-Anastomose $(n=12$, $43 \%)$ oder direkte Naht $(n=7,25 \%)$ des Gallengangs, bei 9 (32\%) Patienten bereits initial durch Anlage einer biliodigestiven Anastomose. Bei 26 (41\%) Patienten traten während des Follow-ups von 96 (12-245) Monaten Komplikationen an den Gallenwegen auf. Bei 9 (14\%) Patienten wurden nach einem Intervall von 22 (8-38) Monaten operative Revisionen $(n=5)$ oder perkutane Dilatationen bei Strikturen $(n=4)$ notwendig. Ein Patient starb 3 Jahre postoperativ an einer biliären Sepsis. 71\% der Patienten hatten ein gutes, 29\% der Patienten ein schlechtes Outcome. Das Vorliegen einer VBV und das Auftreten einer Sepsis nach der initialen Cholezystektomie waren signifikant mit schlechtem Outcome und frühzeitigem Therapieversagen assoziiert. Die Höhe der Gallengangsverletzung und ein initial extern erfolgter Re-
Gallenwegsverletzungen sind schwerwiegende Komplikationen, die häufig mit schlechten Ergebnissen im Langzeitverlauf verbunden sind. Eine VBV und das Auftreten einer Sepsis nach der Cholezystektomie sind Risikofaktoren für ein schlechtes Outcome.

Für die Praxis lassen sich folgende Empfehlungen ableiten:

- Das erste Ziel ist die Vermeidung einer Gallenwegsverletzung im Rahmen der Cholezystektomie.

- Bei der operativen Versorgung einer Gallenwegsverletzung ist die Indikation zur Anlage einer biliodigestiven Anastomose großzügig zu stellen, weil die direkte Naht häufiger zum Therapieversagen führt.

- VBV sind Komplexverletzungen, die häufig mit schlechtem Outcome assoziiert sind (bei Sarno et al. in 67\%) und daher in Zentren für hepatobiliäre Chirurgie versorgt werden sollten.

\section{Korrespondenzadresse}

PD Dr. 0. Strobel

Klinik für Allgemein-, Viszeral- und

Transplantationschirurgie,

Universität Heidelberg,

Im Neuenheimer Feld 110, 69120 Heidelberg

Oliver.Strobel@med.uni-heidelberg.de

Interessenkonflikt. Keine Angaben 